\title{
基于导向策略的钯催化间位 $\mathrm{C}\left(\mathrm{sp}^{2}\right)$ - $\mathrm{H}$ 活化反应的研究进展
}

\author{
袁逸之宋颂焦宁* \\ (北京大学天然药物及仿生药物国家重点实验室 北京 100191)
}

\begin{abstract}
摘要 近些年来, 基于导向策略的 $\mathrm{Pd}$ 催化间位 $\mathrm{C}\left(\mathrm{sp}^{2}\right)-\mathrm{H}$ 键活化反应取得了重要进展. 人们通过远程导向策略, 针对 不同底物发展了多种 U 型导向基, 在一些单保护氨基酸配体(MPAA)的协助下, 实现了多种底物的烯基化、芳基化和乙 酰氧基化反应. 最近, 人们又将 $\mathrm{Pd}$ 催化导向邻位 $\mathrm{C}-\mathrm{H}$ 键活化和 Catellani 反应相结合, 使用结构简单的导向基实现了 高选择性的间位 $\mathrm{C}-\mathrm{H}$ 键的烷基化和芳基化反应. 这两种策略均可用于一些生物活性分子或者药物分子前体的后期修 饰与合成当中. 本文将针对这一领域的最新研究进展做一概述.
\end{abstract}

关键词 钯; 间位 $\mathrm{C}-\mathrm{H}$ 键活化; 远程导向; Catellani 反应; 降冰片烯

\section{Recent Development in $\mathrm{Pd}-$ Catalyzed meta-C(sp $\left.{ }^{2}\right)-\mathrm{H}$ Bond Activation Based on Directing Strategy}

\author{
Yuan, Yizhi Song, Song Jiao, Ning* \\ (School of Pharmaceutical Sciences, State Key Laboratory of Natural and Biomimetic Drugs, \\ Peking University, Beijing 100191)
}

\begin{abstract}
In recent years, remarkable progress has been achieved in Pd-catalyzed meta-C( $\left.\mathrm{sp}^{2}\right)-\mathrm{H}$ bond activation with directing groups. Based on remote activation strategy, a series of U-shaped directing groups have been devised by Yu and others and have been utilized in the olefination, arylation and acetoxylation of different types of substrates with the assistance of mono-protected amino acids (MPAA). Very recently, Yu reported the Pd-catalyzed meta-C( $\left.\mathrm{sp}^{2}\right)-\mathrm{H}$ bond activation via the tandem reaction of ortho- $\mathrm{C}\left(\mathrm{sp}^{2}\right)-\mathrm{H}$ bond activation and Catellani reaction using simple directing groups, in which alkylation and arylation could all be realized under the $\mathrm{Pd} /$ norbornene catalytic system. Dong and co-workers developed arylation of the meta-C( $\left.\mathrm{sp}^{2}\right)-\mathrm{H}$ bond of $N, N$-dimethylbenzylamine with similar strategy. It is noteworthy that these above mentioned meta-C $\left(\mathrm{sp}^{2}\right)-\mathrm{H}$ bond activation protocols could be applied in the late-stage modification and the synthesis of some biologically active molecules and pharmaceutical intermediates. This manuscript will highlight the latest advances in Pd-catalyzed meta-C( $\left(\mathrm{sp}^{2}\right)-\mathrm{H}$ bond activation with directing groups.

Keywords palladium; meta-C-H bond activation; remote direction; Catellani reaction; norbornene
\end{abstract}

在传统的过渡金属催化的偶联反应中, 人们需要利 用预活化的底物才能顺利进行转化, 但往往伴随着大量 卤化物等有机废物的生成. 摆脱预活化底物的使用, 直 接从 $\mathrm{C}-\mathrm{H}$ 键出发构建碳碳键或碳杂原子键无疑是一个 理想的策略. 但由于 $\mathrm{C}-\mathrm{H}$ 键广泛存在于有机分子中, 故实现 $\mathrm{C}-\mathrm{H}$ 键的选择性活化将是首先需要解决的问 题. 经过几十年的迅猛发展, $\mathrm{C}-\mathrm{H}$ 键活化领域已取得了 长足的进步. 人们通过引入导向基团实现了一系列邻位 $\mathrm{C}\left(\mathrm{sp}^{2}\right)-\mathrm{H}$ 键的选择性官能团化. 然而, 导向基间位的 $\mathrm{C}\left(\mathrm{sp}^{2}\right)-\mathrm{H}$ 键的选择性官能团化仍是一个巨大的挑战.

在已有的芳烃间位 $\mathrm{C}\left(\mathrm{sp}^{2}\right)-\mathrm{H}$ 键活化反应中, 人们通 常利用底物固有的位阻 ${ }^{[1]}$ 或电性效应 ${ }^{[2]}$ 调控 $\mathrm{C}-\mathrm{H}$ 键官能 团化的选择性, 因此很大程度上限制了这些反应底物适
用范围. 在传统的 $\mathrm{Pd}$ 催化导向邻位 $\mathrm{C}\left(\mathrm{sp}^{2}\right)-\mathrm{H}$ 键活化反 应中, 需要形成较为刚性的五元或六元环钯中间体. 而 活化间位 $\mathrm{C}\left(\mathrm{sp}^{2}\right)-\mathrm{H}$ 键则需要形成更不稳定的环芳烃类 型的环钯物种, 因此间位 $\mathrm{C}-\mathrm{H}$ 键活化极具挑战性(图 1).

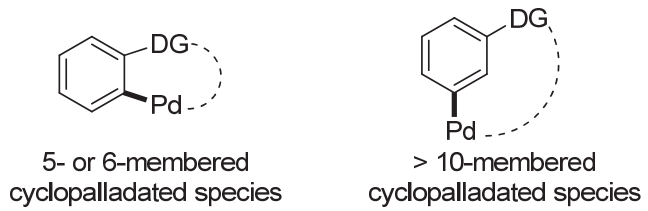

图 1 邻位 $\mathrm{C}-\mathrm{H}$ 键活化和间位 $\mathrm{C}-\mathrm{H}$ 键活化的环钯物种中间体 $(\mathrm{DG}=$ directing group $)$

Figure 1 Cyclopalladated intermediates in ortho- $\mathrm{C}-\mathrm{H}$ bond activation and $m e t a-\mathrm{C}-\mathrm{H}$ bond activation $(\mathrm{DG}=$ directing group $)$

* E-mail: jiaoning@bjmu.edu.cn; Tel.: 0086-010-82805297; Fax: 0086-010-82805297

Received May 10, 2015; published June 1, 2015.

Project supported by the National Basic Research Program of China (973 Program, No. 2015CB856600), the National Natural Science Foundation of China (Nos. 21325206, 21172006), the National Young Top-notch Talent Support Program and the Programs Foundation of the Ministry of Education of China (No. 20120001110013).

项目受国家重点基础研究发展计划(973 计划, No. 2015CB856600)、国家自然科学基金(Nos. 21325206, 21172006)、青年拔尖人才支持计划和国家教育 部博士点基金(No. 20120001110013)资助. 
余金权课题组突破传统间位 $\mathrm{C}-\mathrm{H}$ 键活化的局限性, 利用远程导向策略，设计并合成了两种包含氰基的 $U$ 型 导向基, 开创性地实现了 $\mathrm{Pd}$ 催化芳烃间位 $\mathrm{C}-\mathrm{H}$ 键的烯 基化反应 $^{[3]}$. 反应过程中形成的环钯中间体达到了 11 至 12 元环. 利用这两种 $\mathrm{U}$ 型导向基, 多种 $\alpha, \beta$-不饱和羰基化 合物与芳环间位 $\mathrm{C}-\mathrm{H}$ 键的偶联反应得以实现. 当导向 基团邻位存在苯环结构时，该策略还可以实现联苯骨架 的间位 $\mathrm{C}-\mathrm{H}$ 键烯基化. 基于这一巧妙的间位导向策略, 他们还完成了药物分子 Baclofen 的后期修饰(图 2).
随后，余金权课题组延续这一远程导向的思路，陆 续设计出了多种含有氰基的 $\mathrm{U}$ 型导向基团，搭配该小组 常用的单保护氨基酸配体, 实现了苯酚 ${ }^{[4 a, 4 b]}$ 、氢化肉桂 酸 $^{[4 \mathrm{~b}] \text { 、苯胺 }}{ }^{[4 \mathrm{c}]}$ 、苄胺 ${ }^{[4 \mathrm{c}]}$ 、吲哚啉 ${ }^{[4 \mathrm{~d}]}$ 、苯乙酸类衍生物 ${ }^{[4 \mathrm{e}]}$ 间位 $\mathrm{C}\left(\mathrm{sp}^{2}\right)-\mathrm{H}$ 键的烯基化、芳基化和乙酰氧基化反应. 受到余金权开创性研究工作的启发, $\operatorname{Tan}^{[4 \mathrm{f}]}$ 和 $\mathrm{Maiti}^{[4 \mathrm{~g}]}$ 两 个课题组也发展了两种远程导向基，实现了间位 $\mathrm{C}-\mathrm{H}$ 键的烯基化反应(图 3).<smiles>[R]C=C[Na]</smiles><smiles>[R]C=C([R])c1cccc(COc2c(C(C)(C)C)cccc2C(Br)(C#N)CBr)c1</smiles>

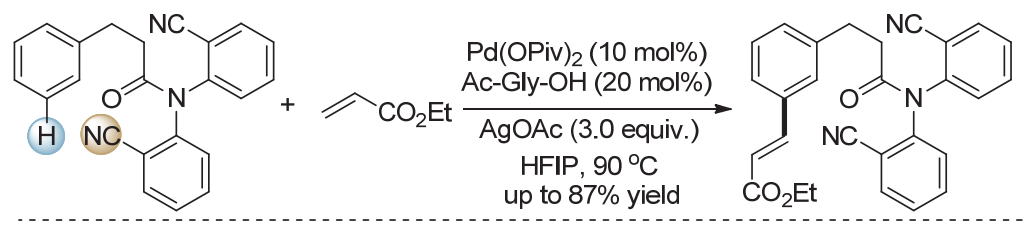<smiles>N#Cc1ccccc1N(C(=O)c1ccccc1-c1ccccc1)c1ccccc1</smiles><smiles>Cc1ccccc1NC(=O)CC(CNNCCO)c1ccccc1</smiles>

(H) NC

Novel biphenyl

Baclofen modification $45 \%$ mono-olefination $40 \%$ mono-olefination $48 \%$ di-olefination $42 \%$ di-olefination

图 2 首例基于远程导向策略的 $\mathrm{Pd}$ 催化间位 $\mathrm{C}-\mathrm{H}$ 键活化反应

Figure 2 The first example of Pd-catalyzed meta-C $-\mathrm{H}$ bond activation based on remote directing strategy

Yu's work<smiles>Cc1cccc(OC(=O)C(C)(C)C)c1</smiles>

H

Boc-Gly-OH/Ac-Gly-OH Olefination/Arylation<smiles>[R]c1cc([R17])c(C(Br)(C#N)C(C)(C)C)cc1[N+](=O)N1CCc2ccccc21</smiles>

Ac-Gly-OH

Olefination/Arylation/ Acetoxylation<smiles>O=C(I)CCc1cccc(O)c1</smiles>

H

Ac-Gly-OH Arylation<smiles>CN1CCCc2ccc(Cc3ccccc3)cc21</smiles>

(H)

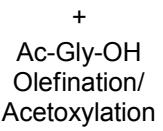

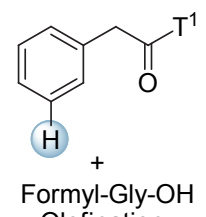
Olefination<smiles>[Y]N1CCCCC1c1cccc(Oc2ccccc2)c1</smiles>

(H)

Ac-Gly-OH Acetoxylation<smiles>C=NN(c1ccccc1C#N)c1ccccc1C#N</smiles><smiles>CC(=O)C(F)Oc1ccccc1N=[I+]</smiles>

Maiti's work

Ac-Gly-OH Olefination<smiles></smiles>

图 3 运用 $\mathrm{U}$ 型导向基导向的其他底物类型的间位 $\mathrm{C}-\mathrm{H}$ 键官能团化

Figure 3 Meta-C-H Bond activation of other types of substrates with U-shaped directing groups 
如上所述, 通过远程活化策略, 人们已经实现了多 种底物的高选择性间位 $\mathrm{C}-\mathrm{H}$ 键官能团化反应. 然而, 该策略最大的不足之处在于需要预先将远程导向基引 入至底物当中, 且导向基的结构复杂, 往往需要多步合 成. 因此, 发展更为简洁高效的间位 $\mathrm{C}-\mathrm{H}$ 键活化策略 仍尤为重要.

余金权课题组基于 Catellani 反应 ${ }^{[5]}$, 发展了一种新 颖的芳烃间位 $\mathrm{C}-\mathrm{H}$ 键活化方式 ${ }^{[6]}$. 传统的 Catellani 反 应利用降冰片烯( $\mathrm{NBE}$ ) 对 $\mathrm{C}-\mathrm{Pd}$ 键的插入反应和 $\beta$-碳消 除反应，可以实现芳基卤化物中碳卤键及其邻位 $\mathrm{C}-\mathrm{H}$ 键的双官能团化 (图 4). 利用 Catellani 反应中间体, Lautens $^{[7 \mathrm{a} \sim 7 \mathrm{e}]}$, Cárdenas 和 Echavarren ${ }^{[7 \mathrm{f}]}$, 董广涁 ${ }^{[7 \mathrm{~g}]}$ 以及 顾振华 ${ }^{[7 \mathrm{~h}, 7 \mathrm{i}]}$ 等课题组分别实现了一系列芳基卤化物的偶 联环化反应. Bach 等 ${ }^{[8]}$ 实现了吲哚和吡咯 2-位 $\mathrm{C}-\mathrm{H}$ 键 的直接官能团化反应.

余金权等巧妙地将 Catellani 反应与导向基团促进 的 $\mathrm{C}-\mathrm{H}$ 键活化相结合, 成功实现了一系列苯乙酸类衍 生物间位 $\mathrm{C}-\mathrm{H}$ 键的烷基化以及芳基化反应(图 5). 该反 应中, 吡啶类配体的使用有效地抑制了其他副反应，大 大提高了间位 $\mathrm{C}-\mathrm{H}$ 键官能团化的效率.

通过对反应机理的分析, 可以预测该反应可能会发 生三种副反应. 首先, 过去已经有较多关于多氟取代苯 乙酰胺类底物的邻位 $\mathrm{C}-\mathrm{H}$ 键活化的报道 ${ }^{[9]}$, 故中间体 I 可能直接发生邻位 $\mathrm{C}-\mathrm{H}$ 键的官能团化; 其次, 中间体 II 可能发生还原消除生成苯并环丁烯类副产物; 第三, 中间体 III 可能再次发生官能团化，得到类似 Catellani 反应的产物. 有趣的是, 作者发现刚性较强的富电子吡
啶配体可以提高目标产物的产率和选择性. 当使用碘甲 烷和碘代乙酸乙酯作为亲电试剂时，各种官能团及多种 环状骨架的底物都可以顺利发生转化. 值得一提的是, 多种生物活性分子衍生物也可以很好地兼容反应体系. 在相同的条件下，间位苄基化反应也得以实现. 将反应 溶剂由 1,2-二氯乙烷 (DCE) 替换为叔丁基甲基醚 (TBME), 即可得到间位芳基化产物, 然而目前只有邻 位吸电子取代的芳基碘代物能兼容反应. 作者认为，这 可能是由于具有配位效应的邻位吸电子基可以促进芳 基钯中间体对芳基碘代物的氧化加成.

董广涁课题组同样以 Catellani 反应为出发点实现 了 $\mathrm{Pd}$ 催化的导向间位 $\mathrm{C}-\mathrm{H}$ 键活化 ${ }^{[10]}$. 作者以 $\mathrm{AsPh}_{3}$ 为配体, $\mathrm{AgOAc}$ 为氧化剂, 同时加入由多种醋酸盐及醋 酸组成的 “醋酸鸡尾酒”，即可以最高 $80 \%$ 的收率得到 $N, N$-二甲基芐胺间位芳基化的产物(图 6). 有趣的是，当 缺少 “醋酸鸡尾酒” 中的任何一种组分时, 产率都会发 生不同程度的下降. 同样, 该反应目前也只能以邻位吸 电子基取代的芳基碘代物为芳基化试剂. 当 $N, N$-二甲基 苄胺的两个间位均无取代基时，反应生成双芳基化产 物.

综上所述，目前基于导向策略的 $\mathrm{Pd}$ 催化间位 $\mathrm{C}-\mathrm{H}$ 键活化反应大体分为两类: (1)基于远程导向策略的直接 间位 $\mathrm{C}-\mathrm{H}$ 键官能团化; (2)邻位 $\mathrm{C}-\mathrm{H}$ 键活化和 Catellani 反应串联的间位 $\mathrm{C}-\mathrm{H}$ 键官能团化. 经过近几年的发展, 远程导向策略发展较快, 人们目前已经可以实现苄醇 类、胺类、苯乙酸类及氢化肉桂酸类多种底物类型的间

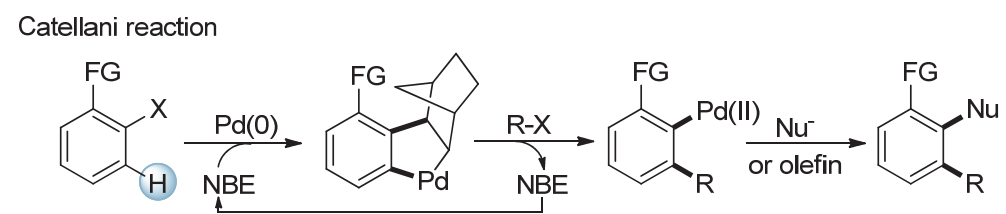

图 4 Catellani 反应中碳卤键的邻位和原位官能团化

Figure 4 The ortho- and ipso-functionalization in the Catellani reaction

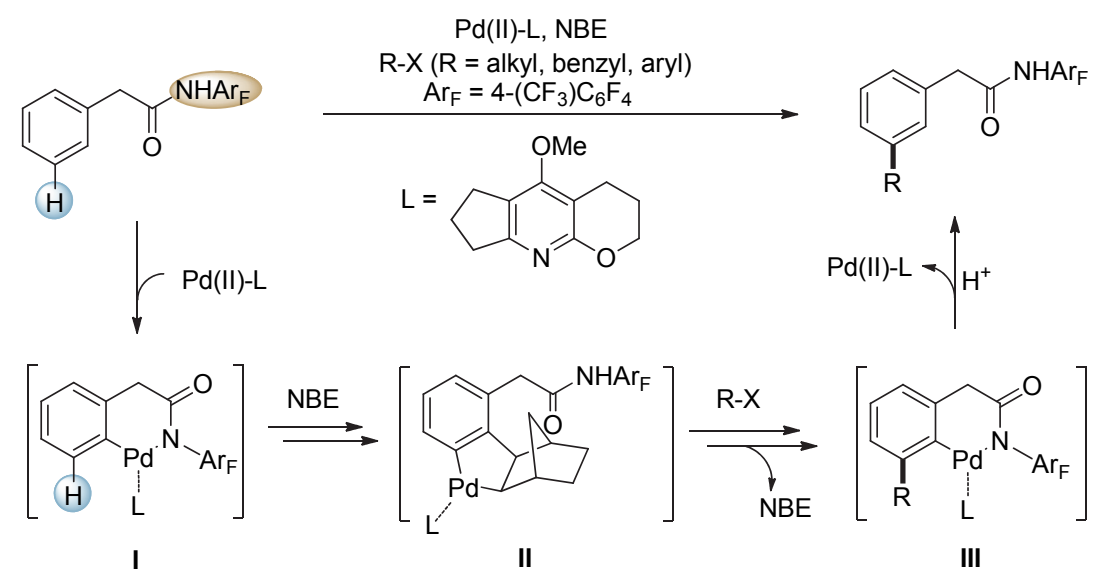

图 5 基于 Catellani 反应的 $\mathrm{Pd}$ 催化导向邻位 $\mathrm{C}-\mathrm{H}$ 键官能团化

Figure 5 Pd-catalyzed directed meta-C- $-\mathrm{H}$ bond functionalization based on Catellani reaction 


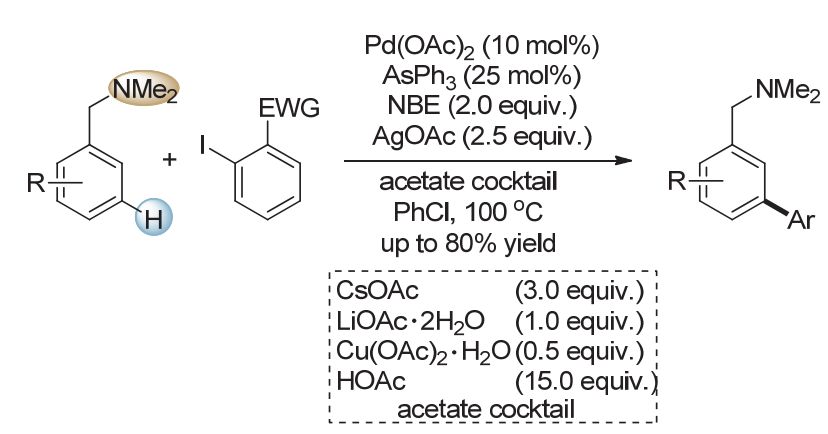

图 6 基于 Catellani 反应的 $\mathrm{Pd}$ 催化导向芐胺类间位 $\mathrm{C}-\mathrm{H}$ 键官能团化 Figure 6 Pd-catalyzed directed meta-C-H bond functionalization of benzylamine based on Catellani reaction

位 $\mathrm{C}-\mathrm{H}$ 键的烯基化、芳基化和乙酰氧基化. 而基于 Catellani 策略的间位 $\mathrm{C}-\mathrm{H}$ 键活化才刚刚起步. 因此, 该领域目前还有很多方面值得进一步探索. 例如: (1)发 展更加简单、更加实用的导向基; (2)通过导向基和配体 的合理设计实现更好的间位选择性; (3)完善导向基设计 的指导原则; (4)发展其他过渡金属催化的导向间位 C$\mathrm{H}$ 键活化反应等等.

\section{作者简介}

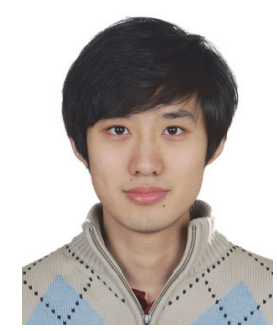

袁逸之, 1991 年出生于山东, 2013 年于北京大学药学院取 得学士学位, 同年加入北京大学天然药物及仿生药物国家重 点实验室焦宁教授课题组取得硕士学位. 主要研究方向为叠 氮参与的含氮杂环的构建.

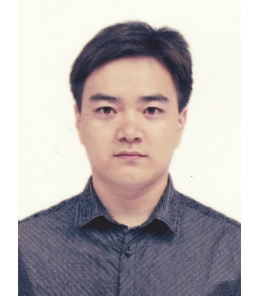

宋颂, 1985 年出生于江苏, 于 2007 年和 2012 年在南开大 学化学院取得学士学位和博士学位(导师: 周其林院士). 2013
年至今在北京大学药学院焦宁课题组从事博士后研究工作. 主要研究方向为基于二甲基亚砜为氧化剂的氧化反应研究.

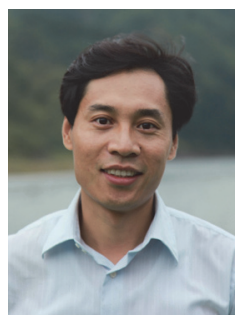

焦宁教授 1976 年出生于山东, 1999 年在山东大学化学系 取得学士学位. 1999 年至 2004 年在中国科学院上海有机化学 研究所学习并取得博士学位(导师：麻生明院士). 2004 年至 2006 年在德国马普煤炭研究所 Manfred T. Reetz 教授课题组从 事博士后研究工作. 2007 年加入北京大学天然药物及仿生药 物国家重点实验室. 其主要研究兴趣是: (1)基于单电子转移过 程的绿色合成方法学; (2)需氧氧化; 氧合反应; 氮化反应; (3) 惰性化学键的活化; (4)小分子活化.

\section{References}

[1] Cho, J.-Y.; Tse, M. K.; Holmes, D.; Maleczka, R. E. Jr.; Smith, M. R. III. Science 2002, 295, 305.

[2] (a) Zhang, Y.-H.; Shi, B.-F.; Yu, J.-Q. J. Am. Chem. Soc. 2009, 131, 5072. (b) Ye, M.; Gao, G.-L.; Yu, J.-Q. J. Am. Chem. Soc. 2011, 133, 6964.

[3] Leow, D.; Li, G.; Mei, T.-S.; Yu, J.-Q. Nature 2012, 486, 518.

[4] (a) Dai, H.-X.; Li, G.; Zhang, X.-G.; Stepan, A. F.; Yu, J.-Q. J. Am. Chem. Soc. 2013, 135, 7567. (b) Wan, L.; Dastbaravardeh, N.; Li, G.; Yu, J.-Q. J. Am. Chem. Soc. 2013, 135, 18056. (c) Tang, R.-Y.; Li, G.; Yu, J.-Q. Nature 2014, 507, 215. (d) Yang, G.; Lindovska, P.; Zhu, D.; Kim, J.; Wang, P.; Tang, R.-Y.; Movassaghi, M.; Yu, J.-Q. J. Am. Chem. Soc. 2014, 136, 10807. (e) Deng, Y.; Yu, J.-Q. Angew. Chem., Int. Ed. 2015, 54, 888. (f) Lee, S.; Lee, H.; Tan, K. L. J. Am. Chem. Soc. 2013, 135, 18778. (g) Bera, M.; Modak, A.; Patra, T.; Maji, A.; Maiti, D. Org. Lett. 2014, 16, 5760.

[5] Catellani, M.; Frignani, F.; Rangoni, A. Angew. Chem., Int. Ed. Engl. 1997, 36, 119.

[6] Wang, X.-C.; Gong, W.; Fang, L.-Z.; Zhu, R.-Y.; Li, S.; Engle, K. M.; Yu, J.-Q. Nature 2015, 519, 334.

[7] (a) Bressy, C.; Alberico, D.; Lautens, M. J. Am. Chem. Soc. 2005, 127, 13148. (b) Mariampillai, B.; Alliot, J.; Li, M.; Lautens, M. J. Am. Chem. Soc. 2007, 129, 15372. (c) Gericke, K. M.; Chai, D. I; Bieler, N.; Lautens, M. Angew. Chem., Int. Ed. 2009, 48, 1447. (d) Candito, D. A.; Lautens, M. Angew. Chem., Int. Ed. 2009, 48, 6713. (e) Zhao, Y.-B.; Mariampillai, B.; Candito, D. A.; Laleu, B.; Li, M.; Lautens, M. Angew. Chem., Int. Ed. 2009, 48, 1849. (f) Cárdenas, D. J.; Martín-Matute, B.; Echavarren, A. M. J. Am. Chem. Soc. 2006, 128, 5033. (g) Dong, Z.; Dong, G. J. Am. Chem. Soc. 2013, 135, 18350. (h) Sui, X.; Zhu, R.; Li, G.; Ma, X.; Gu, Z. J. Am. Chem. Soc. 2013, 135, 9318. (i) Sun, F.; Gu, Z. Org. Lett. 2015, 17, 2222.

[8] (a) Jiao, L.; Bach, T. J. Am. Chem. Soc. 2011, 133, 12990. (b) Jiao, L.; Herdtweck, E.; Bach, T. J. Am. Chem. Soc. 2012, 134, 14563. (c) Jiao, L.; Bach, T. Angew. Chem., Int. Ed. 2013, 52, 6080.

[9] Engle, K. M.; Mei, T.-S.; Wasa, M.; Yu, J.-Q. Acc. Chem. Res. 2012, 45, 788 .

[10] Dong, Z.; Wang, J.; Dong, G. J. Am. Chem. Soc. 2015, 137, 5887.

(Zhao, C.) 